\title{
Zur Theorie der Supraleitung
}

\author{
Von Werner Heisenberg \\ Aus dem Max-Planck-Institut der Kaiser-Wilhelm-Gesellschaft, Göttingen \\ (Z. Naturforschg, 2 a, 185-201 [1947]; eingegangen am 20. September 1946)
}

\begin{abstract}
Durch die Überlegungen in Kap. 1 wird zunächst festgestellt, daß die Coulombsche Abstoßung das Verhalten des „Fermi-Gases“ der Leitungselektronen im Metall entscheidend beeinflußt. Zwar spielt diese Abstoßung keine allzu wichtige Rolle für die Elektronen, deren Energie kleiner ist als die Grenzenergie des Fermi-Gases. Aber gerade die Elektronen mit der Grenzenergie, also die relativ wenigen Elektronen, die für die spezifische Wärme maßgebend sind, werden durch die lange Reichweite der Coulomb-Kräfte so stark in ihrem Verhalten beeinflußt, daß für sie das FermiGas keine Approximation mehr ist und daß hier mit einer Ordnung auf weite Abstände gerechnet werden muß. Für diese Ordnung wird in Kap. 2 ein Modell versucht, dessen mathematische Durchführung allerdings noch unbefriedigend ist; aber vielleicht treffen die qualitativen Züge dieses Modells (logarithmische Abhängigkeit der potentiellen Energie von der Dichte der geordneten Phase usw.) schon das Richtige. In den Kap. 3 und 4 wird dann gezeigt, daß das thermodynamische und das elektrische Verhalten eines Supraleiters von einem solchen Modell aus qualitativ verstanden werden kann. Wenn diese Utberlegungen, die im ganzen mehr ein Programm als eine Theorie sind, die Ursachen für die Supraleitung an der richtigen Stelle suchen, so sollte die Supraleitung ein ganz allgemeines Phänomen bei allen echten Elektronenleitern sein und nicht an sehr spezielle Bedingungen, wie etwa innere $d$ - oder f-Zustände (so wie beim Ferromagnetismus) und dergl., geknüpft sein. Jedoch ist zu erwarten, daß die Sprungtemperaturen verschiedener Leiter sich eventuell um mehrere, vielleicht manchmal viele Größenordnungen unterscheiden; die Sprungtemperaturen sollten um so höher liegen, je dichter die maßgebenden Energiebänder mit Elektronen besetzt sind (d. h. je schmäler die Bänder sind); also gerade relativ schlechte Leiter sollten hohe Sprungpunkte haben. Ferromagnetische Metalle sollten nicht supraleitend werden können, da die Ordnung der supraleitenden Phase nach dieser Theorie auf der Coulo mbschen Wechselwirkung von Elektronen entgegengesetzter Spinrichtung beruht. Der Meißner-OchsenfeldEffekt ist nach den hier beschriebenen Überlegungen eine Folge des zweiten Hauptsatzes und deutet nicht auf irgendeine besondere Beziehung zwischen Supraleitung und Diamagnetismus hin.
\end{abstract}

W ährend die meisten Eigenschaften der Materie im großen durch die Atomtheorie wenigstens qualitativ erklärt werden können, fehlt für das Phänomen der Supraleitung bisher eine befriedigende Deutung. Zwar geben die von F. L o n d o $\mathrm{n}^{\mathbf{1}}$ aufgestellten und von M. v. L a u e ${ }^{2}$ ausführlich behandelten Gleichungen eine ausreichende phänomenologische Beschreibung des elektrodynamischen Verhaltens der Supraleiter, und auch die thermodynamische Seite des Problems ist durch die Arbeiten von Gorter ${ }^{3}$ im

1 F. L on d on, Une conception nouvelle de la supraconductibilité, Paris 1937; Nature [London] 140, 796, 834 [1937].

2 M. v. Lau e, Ann. Physik (5) 32, 71, 253 [1938]. wesentlichen geklärt worden. Aber es gibt bisher keine befriedigende atomtheoretische Erklärung für das Phänomen selbst, es ist nicht einmal möglich gewesen, ein Modell sich auszudenken, das mit Sicherheit die Eigenschaften des Supraleiters besäße. R. de Lar Kronig ${ }^{4}$ hat schon vor längerer Zeit den Gedanken ausgesprochen, daß die Elektronen sich im Supraleiter zu einer Art Kristallgitter ordnen könnten und daß ein solches bewegtes Gitter keinen Widerstand im Metall erführe; aber er hat diesen Gedanken nicht wirk-

3 C. J. Gorter, Nature [London] 132, 931 [1933]; C. J. Gorter u. H. Casim ir, Physica 1, 306 [1934]. 4 R. de L. K r on i g, Z. Physik 78, 744 [1932]; 80, 203 [1932]. 
lich durchführen können und später angenommen, daß es sich um die Ordnung in linearen Ketten handeln soll. H. Welker ${ }^{5}$ hat im Anschluß an L o n d o ${ }^{1}$ versucht, von der Ähnlichkeit des Supraleitungsstromes mit dem für den Diamagnetismus maßgebenden Strom auszugehen. Tatsächlich würde man die Supraleitung verstehen können, wenn es irgendein Modell gäbe, bei dem sich die Wellenfunktion bei Einschalten eines Magnetfeldes nicht merklich ändert. Dann würde nämlich in dem üblichen Ausdruck für den Strom

$$
\begin{aligned}
\mathfrak{S}=-\frac{i \hbar e}{2 m \cdot}\left\{\psi^{*} \operatorname{grad} \psi-\psi \operatorname{grad} \psi^{*}\right\} & \\
& -\frac{e^{2}}{m c} \mathfrak{A} \psi^{*} \psi
\end{aligned}
$$

nur der zweite Teil einen Beitrag liefern, und der hängt vom Magnetfeld genau in der von L ond on geforderten Form ab. Aber auch Welker hat kein Modell angeben können, das diese Eigenschaft sicher besitzt; er hat angenommen, daß magnetische Austauschkräfte unter Umständen diese Wirkung hervorbringen können.

Auch die vorliegende Arbeit kann keine wirklich ausgearbeitete Lösung für das offenbar sehr schwierige Problem der Supraleitung vorschlagen; sie beabsichtigt vielmehr nur, auf einen bestimmten Zug in der Theorie der metallischen Leiter hinzuweisen, der bisher übersehen worden ist, und der vielleicht die Stelle bezeichnet, wo man nach einer Erklärung für die Supraleitung suchen soll. Dabei soll von der folgenden Überlegung ausgegangen werden: Die Leitungselektronen im Metall können in einer gewissen Näherung als ein Gas aufgefaßt werden, das der Fermi-Statistik genügt. Diese Elektronen erfüllen im Impulsraum näherungsweise eine Kugel, wenn nur ein relativ kleiner Bruchteil des betreffenden Energiebandes aufgefüllt ist, oder sie lassen näherungsweise eine Kugel von „Löchern“ übrig, wenn das Energieband nahezu aufgefüllt ist. Bei den tiefen Temperaturen, bei denen Supraleitung eintreten kann, spielen sich alle Vorgänge nur in einer dünnen Oberflächenschicht dieser Impulskugel ab. Denn die weiter innen liegenden Elektronen können wegen des PauliPrinzips keine Übergänge mit kleinen Energieänderungen ausführen, da die umgebenden $\mathrm{Zu}$ stände besetzt sind, und Ǔbergänge mit großen

5 H. Welk er, Physik. Z. 39, 920 [1938].
Energieänderungen kommen bei tiefen Temperaturen praktisch nicht vor. Dies gilt z. B. für die spezifische Wärme oder für die gewöhnliche elektrische Leitung, und man kann abschätzen, daß bei $1^{\circ} \mathrm{K}$ nur noch etwa der $10^{4}$-te Teil aller Leitungselektronen an der spezifischen Wärme wirklich beteiligt ist. Da die Energiebeträge, die beim Ǔbergang in die supraleitende Phase frei werden, ebenfalls sehr klein sind, kann auch die Supraleitung nur mit einer Besonderheit im Verhalten dieses minimalen Bruchteils aller Leitungselektronen zusammenhängen. Alle Elektronen in der dünnen Oberflächenschicht der Impulskugel besitzen praktisch den gleichen, von Null verschiedenen Absolutwert des Impulses und damit die gleiche absolute Geschwindigkeit. Sobald nun durch irgendwelche Wechselwirkungen eine gegenseitige Ordnung dieser Elektronen im Koordinatenraum hervorgerufen wird, liegt es nahe, anzunehmen, daß auch dieses geordnete System von Elektronen eine gemeinsame Geschwindigkeit und damit einen Strom besitzt. Möglicherweise kann also durch die Wechselwirkung erreicht werden, daß der thermodynamisch stabilste $\mathrm{Zu}$ stand bei tiefen Temperaturen eben ein solcher geordneter Zustand mit Strom ist, was natürlich zu Vorgängen Anlaß geben müßte, die denen bei der Supraleitung zum mindesten sehr ähnlich sind. Daher entsteht die Aufgabe, zu untersuchen, ob nicht die Elektronen an der Oberfläche der Impulskugel durch ihre Wechselwirkung in einen geordneten Zustand übergehen können. Die bei weitem stärkste Wechselwirkung ist die elektrostatische Abstoßung der Elektronen; deren Wirkung soll daher im folgenden vor allem untersucht werden.

1. Die Coulombsche Wechselwirkung im Fermi-Gas der Leitungselektronen

a) Die Ladung der Leitungselektronen gibt, zusammen mit der Ladung der übrigbleibenden Ionen, Anlaß zu einem mittleren elektrischen Feld, das in der üblichen Weise aus der Gleichung $\operatorname{div} \tilde{\xi}=4 \pi \varrho$ bestimmt wird, wobei $\varrho$ die mittlere Ladungsdichte bedeutet, die von Elektronen und Ionen hervorgerufen wird und in der Regel (zum mindesten im Mittel) verschwindet. Dieses mittlere. Feld wird durch die elektrodynamischen Gleichungen richtig berücksichtigt und kann nicht Anlaß zu irgendwelchen Ordnungsphänomenen 
geben. Anders ist es aber mit der Abweichung des Feldes vom Mittelwert. Diese Abweichung kann die Wellenfunktion des Systems so beeinflussen, daß die Wahrscheinlichkeit, ein Elektron in der Nachbarschaft eines anderen zu finden, verringert wird, und durch diesen Effekt kann eine gewisse Ordnung der Elektronen hervorgerufen werden. Allerdings wird man diese Ordnung vielleicht nicht im gewöhnlichen Raum - etwa als Elektronengitter - beschreiben können, vielmehr wird gerade die Kopplung der Elektronen im Konfigurationsraum für dieses Ordnungsphänomen charakteristisch sein.

Um einen Anhalt dafür zu bekommen, in welcher Richtung die Abweichung der Wellenfunktion von der des einfachen F e r m i - Gases liegen kann, werden wir eine Störungsrechnung durchführen, in der das Fe rmi-Gas am absoluten Nullpunkt als Ausgangslösung verwendet wird und in der die Coulomb-Kraft als kleine Störung betrachtet werden soll. Allerdings wird sich dabei charakteristischerweise herausstellen, daß die Störungsrechnung nicht konvergiert; aber die Art, wie die Störung die Wellenfunktion beeinflußt, kann als Wegweiser für die späteren Untersuchungen dienen.

Wir nehmen für die folgenden Rechnungen an, daß das Energieband der Leitungselektronen nur wenig aufgefüllt ist, so daß die Energie $E$ der Elektronen mit ihrem Impuls $\mathfrak{p}$ durch die übliche Gleichung

$$
E=\mathfrak{p}^{2} / 2 m
$$

verknüpft ist. $m$ bedeutet die für das betreffende Energieband charakteristische effektive Masse der Elektronen. Der Radius $P$ der Kugel im Impulsraum, die von Elektronen erfüllt ist, hängt mit der räumlichen Dichte $n$ der Elektronen durch die Gleichung

$$
n=\frac{8 \pi}{3} \frac{P^{3}}{h^{3}}
$$

zusammen. Die Coulombsche Wechselwirkung gibt zu UUbergangselementen der Störungsenergie Anlaß, bei denen ein Elektron seinen Impuls von $\mathfrak{p}_{1}^{\prime}$ nach $\mathfrak{p}_{1}^{\prime \prime}$, und ein zweites Elektron seinen Impuls um die entgegengesetzt gleiche Differenz von $\mathfrak{p}_{2}{ }^{\prime}$ nach $\mathfrak{p}_{2}{ }^{\prime \prime}$ ändert. Für den Utbergang muß also gelten:

$$
\mathfrak{p}_{1}{ }^{\prime}+\mathfrak{p}_{2}{ }^{\prime}=\mathfrak{p}_{1}{ }^{\prime \prime}+\mathfrak{p}_{2}{ }^{\prime \prime},
$$

und das betreffende Übergangselement lautet:

$$
\begin{aligned}
& \left(\mathfrak{p}_{1}^{\prime}, \mathfrak{p}_{2}{ }^{\prime}|H| \mathfrak{p}_{1}{ }^{\prime \prime}, \mathfrak{p}_{2}{ }^{\prime \prime}\right) \\
& =\iint d \mathfrak{r}_{1} d \mathfrak{r}_{2} \frac{e^{2}}{\left|\mathfrak{r}_{1}-\mathfrak{r}_{2}\right|} e^{\left.\frac{i}{\hbar}\left[\left(\mathfrak{p}_{1}^{\prime \prime}-\mathfrak{p}_{1}^{\prime}\right) \mathfrak{r}_{1}+\left(\mathfrak{p}_{2}{ }^{\prime \prime}-\mathfrak{p}_{2}\right)^{\prime}\right) \mathfrak{r}_{2}\right]} \\
& =h^{3} \delta\left(\mathfrak{p}_{1}{ }^{\prime}+\mathfrak{p}_{2}{ }^{\prime}-\mathfrak{p}_{1}{ }^{\prime \prime}-\mathfrak{p}_{2}{ }^{\prime \prime}\right) \int d \mathfrak{s} \frac{e^{2}}{s} e^{\frac{i}{\hbar}\left(\mathfrak{p}_{1}{ }^{\prime}-\mathfrak{p}_{1}{ }^{\prime \prime}\right) \mathfrak{s}} \\
& =h^{3} \delta\left(\mathfrak{p}_{1}{ }^{\prime}+\mathfrak{p}_{2}{ }^{\prime}-\mathfrak{p}_{1}{ }^{\prime \prime}-\mathfrak{p}_{2}{ }^{\prime \prime}\right) 4 \pi e^{2} \hbar^{2} /\left(\mathfrak{p}_{1}{ }^{\prime}-\mathfrak{p}_{1}{ }^{\prime \prime}\right)^{2}
\end{aligned}
$$

Allerdings hat das Übergangselement nur dann diesen Wert (4), wenn die Zustände $\mathfrak{p}_{1}{ }^{\prime \prime}$ und $\mathfrak{p}_{2}{ }^{\prime \prime}$ außerhalb der Kugel vom Radius $P$ liegen; denn sonst wären diese Zustände besetzt, und die Übergangselemente würden verschwinden. Wenn man die Impulsdifferenz

$$
\mathfrak{p}_{1}^{\prime \prime}-\mathfrak{p}_{1}{ }^{\prime}=\mathfrak{g}
$$

vorgibt, so müssen also $\mathfrak{p}_{1}^{\prime}$ und $\mathfrak{p}_{2}^{\prime}$ in solchen Gebieten der Kugel liegen, daß $\mathfrak{p}_{1}{ }^{\prime}+\mathfrak{g}$ und $\mathfrak{p}_{2}{ }^{\prime}-\mathfrak{g}$ außerhalb derKugel liegen; für sehr kleine Werte von $\mathfrak{g}$ sind also $\mathfrak{p}_{1}{ }^{\prime}$ und $\mathfrak{p}_{2}{ }^{\prime}$ auf eine dünne Oberflächenschicht der Kugel beschränkt.

Wenn man die Energie des Gases im gestörten Zustand berechnen will, so braucht man zunächst die Störungsenergie erster Ordnung, die durch den Mittelwert des Störungsoperators über das ungestörte System gegeben ist. Dieser Mittelwert verschwindet hier, weil das Metall im ganzen elektrisch neutral sein soll. Für das hier gestellte Problem ist also erst die Störungsenergie zweiter Ordnung wichtig, die nach einer bekannten Formel der Quantenmechanik durch den Ausdruck

$$
W_{2}=-\sum_{k} \frac{\left|H_{0 k}\right|^{2}}{E_{k}-E_{0}}
$$

gegeben ist. Hierin bedeutet $H_{0 k}$ das Utbergangselement der Störungsenergie vom Zustand 0 zum Zustand $k, E_{0}$ und $E_{k}$ sind die zugehörigen ungestörten Energiewerte. In (6) wird über alle möglichen angeregten Zustände summiert. Die angeregten Zustände des Gesamtsystems sind in unserem Fall durch drei Impulse charakterisiert: die beiden Impulse $\mathfrak{p}_{1}{ }^{\prime}$ und $\mathfrak{p}_{2}{ }^{\prime}$, denen je ein Elektron ,entzogen“" wird, und den Impuls g, der durch die Beziehungen

$$
\mathfrak{p}_{1}{ }^{\prime \prime}=\mathfrak{p}_{1}{ }^{\prime}+\mathfrak{g} ; \quad \mathfrak{p}_{2}{ }^{\prime \prime}=\mathfrak{p}_{2}{ }^{\prime}-\mathfrak{g}
$$

angibt, welche Impulse nun ein Elektron erworben haben. Im angeregten Zustand $\mathfrak{p}_{1}{ }^{\prime}, \mathfrak{p}_{2}{ }^{\prime}, \mathfrak{g}$ fehlt also in der Fer.mi-Kugel je ein Elektron an den Stellen $\mathfrak{p}_{1}{ }^{\prime}, \mathfrak{p}_{2}{ }^{\prime}$, und es findet sich außerhalb 
der Kugel je eines an den Stellen $\mathfrak{p}_{1}^{\prime}+\mathfrak{g}$ und $\mathfrak{p}_{2}{ }^{\prime}-\mathfrak{g}$.

Der Nenner der Formel (6) ist demnach gegeben durch:

$$
E_{\mathfrak{p}_{1}^{\prime}, \mathfrak{p}_{2}, \mathfrak{g}}-E_{0}=\frac{1}{m}\left[\left(\mathfrak{p}_{1}{ }^{\prime}-\mathfrak{p}_{2}{ }^{\prime}\right) \mathfrak{g}+\mathfrak{g}^{2}\right] .
$$

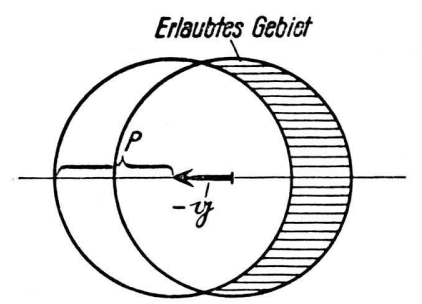

Abb. 1.

Die Summation über $k$ in $(6)$ wird hier eine Integration über die drei Impulse $\mathfrak{p}_{1}{ }^{\prime}, \mathfrak{p}_{2}{ }^{\prime}$, g. Da das Matrixelement (4) nür von g abhängt, führt man die Integrationen über $\mathfrak{p}_{1}{ }^{\prime}$ und $\mathfrak{p}_{2}{ }^{\prime}$ zweckmäßig zuerst aus. Das erlaubte Gebiet für $\mathfrak{p}_{1}^{\prime}$ erhält man, wenn man die Impulskugel vom Radius $P$ zum Schnitt bringt mit einer zweiten Kugel vom gleichen Radius, deren Mittelpunkt gegen die erste $\mathrm{um}-\mathfrak{g}$ verschoben ist (vergl. Abb. 1). Für die Integration ist es zweckmäßig, die zu g parallelen Komponenten von $\mathfrak{p}_{1}{ }^{\prime}$ und $\mathfrak{p}_{2}{ }^{\prime}$ als Variable einzuführen und die zugehörigen erlaubten Flächen im Impulsraum zu berechnen. Der kleinste Wert der Parallelkomponente von $\mathfrak{p}_{1}{ }^{\prime}$ ist offenbar $-\mathfrak{g} / 2$, ebenso ist der größte Wert der Parallelkomponente von $\mathfrak{p}_{2}{ }^{\prime}:+\mathfrak{g} / 2$. Man führt daher zweckmäßig ein :

$$
x=\mathfrak{p}_{1}^{\prime}{ }_{\|}+\mathrm{g} / 2 ; \quad y=-\mathfrak{p}_{2}{ }_{\|}+\mathrm{g} / 2 .
$$

Dann können $x$ und $y$ nur positive Werte annehmen. Die Energiedifferenz (8) wird:

$$
E_{\mathfrak{p}_{1}^{\prime}, \mathfrak{p}^{\prime}, \mathfrak{g}}-E_{0}=\frac{g}{m}(x+y) \text {. }
$$

Man entnimmt aus der Abb. 1, daß die zu $x$ und $y$ gehörigen ,erlaubten“ Flächen $f(x)$ bzw. $f(y)$ die folgenden Werte haben:

$$
f(x)=\left\{\begin{array}{ccc}
2 \pi x g & \text { für } & 0 \leqslant x \leqslant P-g / 2 \\
\pi\left[P^{2}-(x-g / 2)^{2}\right] & \text { für } P-g / 2 \leqslant x \leqslant P+g / 2 \\
0 & \text { für } P+g / 2 \leqslant x .
\end{array}\right.
$$

Die gleichen Beziehungen gelten für $f(y)$.

Zur Ableitung der Störungsenergie (6) führen wir also zunächst die Integration über $\mathfrak{p}_{1}^{\prime}$ und $\mathfrak{p}_{2}{ }^{\prime}$ aus und berechnen das Integral:

$$
I=\int \frac{2 d \mathfrak{p}_{1}{ }^{\prime} \cdot 2 d \mathfrak{p}_{2}{ }^{\prime}}{h^{6}\left(E_{\mathfrak{p}_{1}^{\prime}, \mathfrak{p}_{2}, \mathfrak{g}}-E_{0}\right)}=\frac{4 m}{g h^{6}} \int \frac{d x d y f(x) f(y)}{x+y} .
$$

Die Faktoren 2 in $2 / h^{3}$ rühren davon her, daß jeder Zustand im Inneren der Impulskugel doppelt besetzt ist. Dagegen wird die spätere Integration über $\mathfrak{g}$ nur den Faktor $h^{-3}$ mit sich führen, da die Coulomb-Kraft nur Übergänge vermittelt, bei denen sich der Spin des Elektrons nicht ändert.

Für das Integral (12) findet man nach etwas umständlichen elementaren Rechnungen:

$$
I=\frac{2 m \pi^{2}}{15 g h^{6}}\left\{\begin{array}{r}
{\left[32 P^{5} \lg \left(1-\frac{g^{2}}{4 P^{2}}\right)+g\left(30 P^{4}-5 P^{2} g^{2}+\frac{3}{8} g^{4}\right) \lg \frac{P+g / 2}{P-g / 2}\right.} \\
\left.+58 P^{3} g^{2}-\frac{3}{2} P g^{4}-80 P^{3} g^{2} \lg 2\right] \text { für } g \leqslant 2 P, \\
{\left[g^{3}\left(g^{2}-20 P^{2}\right) \lg \left(1-\frac{4 P^{2}}{g^{2}}\right)+8 P^{3}\left(5 g^{2}-4 P^{z}\right) \lg \frac{g-2}{g+2} \frac{2}{P}\right.} \\
\left.+4 P^{2} g^{3}+88 g P^{4}\right] \text { für } g \geqslant 2 P .
\end{array}\right.
$$

Für sehr kleine und sehr große Werte von $g$ geht (13) über in: 
$I \approx \begin{cases}\frac{32}{3} \frac{m \pi^{2}}{h^{6}} P^{3} g(1-\lg 2) & \text { für } g \leqslant 2 P, \\ \frac{64}{9} \frac{m \pi^{2}}{h^{6}} \frac{P^{6}}{g^{2}} & \text { für } g \geqslant 2 P .\end{cases}$

Das Matrixelement $H_{0 k}$ wird nach (4), etwa durch Integration über $\mathfrak{p}_{2}{ }^{\prime \prime}$ :

$$
H_{0 ; \mathfrak{p}_{\mathfrak{1}}^{\prime} \mathfrak{p}_{2}^{\prime} g}=4 \pi e^{2} \hbar^{2} / g^{2} .
$$

Bezeichnet man die Energiedichte des FermiGases mit $u$, so erhält man schließlich nach (6) als Störungsenergiedichte zweiter Ordnung

$$
u_{2}=\int_{0}^{\infty} \frac{4 \pi g^{2} d g}{h^{3}} I(g) \cdot\left(\frac{4 \pi e^{2} \hbar^{2}}{g^{2}}\right)^{2} .
$$

Dieses Integral divergiert bei kleinen Werten von $g$ logarithmisch, es verhält sich [nach (2), (14) und (16)] dort wie

$$
u_{2} \sim \frac{16 m e^{4}}{h^{2}}(1-\lg 2) n \int \frac{d g}{g} .
$$

Dagegen konvergiert das Integral bei großen Werten von $g$.

Dieses Ergebnis bedeutet zunächst, daß die übliche Wellenfunktion des Fermi-Gases am absoluten Nullpunkt keine brauchbare Approximation für den tiefsten Zustand des Systems darstellt. Die von den Coulombschen Kräften herrührenden Störungen bewirken zwar, soweit sie von den inneren Elektronen der Impulskugel herrühren, nur eine endliche Änderung der Energie; aber die unmittelbar an der Oberfläche der Impulskugel liegenden Elektronen werden durch die auf weite Abstände wirkende Coulomb-Kraft so stark gestört, daß die wahre Wellenfunktion hier offenbar qualitativ von der ungestörten abweicht. Man wird also zu der Vermutung geführt, daß durch die große Reichweite der Coulomb-Kräfte die an der Oberfläche der Impulskugel liegenden Elektronen irgendwie anders geordnet werden können; dabei dürfte es sich nur um eine sehr dünne Oberflächenschicht handeln, da das Integral (17) nur sehr schwach, nämlich logarithmisch, divergiert.

b) Um einen Anhaltspunkt dafür zu bekommen, von welcher Art die Ordnung sein kann, die hier möglicherweise von den Coulomb-Kräften hervorgerufen wird, ist es zweckmäßig, die durch die Störung hervorgerufene Veränderung der Wellenfunktion zu studieren.

Nach bekannten Formeln der Quantenmechanik lautet die Wellenfunktion des gestörten Systems in erster Näherung:

$$
\psi=\psi_{0}-\sum_{k} \frac{H_{k o}}{E_{k}-E_{0}} \psi_{k},
$$

wobei $\psi_{0}$ und $\psi_{k}$ die ungestörten Wellenfunktionen der betreffenden Zustände sind. $\psi_{0}$ und $\psi_{k}$ sind die bekannten antisymmetrischen Wellenfunktionen, die von den Orts- und Spinkoordinaten sämtlicher Elektronen abhängen. Die anschauliche Bedeutung der in (18) angegebenen Veränderung der Wellenfunktion erkennt man am besten, wenn man nach der Wahrscheinlichkeit $w\left(\mathfrak{r}_{1}, \mathfrak{r}_{2}\right)$ fragt, ein Elektron an der Stelle $\mathfrak{r}_{2} \mathrm{zu}$ finden, wenn man weiß, daß ein anderes Elektron an der Stelle $\mathfrak{r}_{1}$ ist. Man muß zu diesem Zweck das Produkt $\psi^{*} \psi$ über alle Ortskoordinaten $\mathfrak{r}_{3}$, $\mathfrak{r}_{4} \ldots$ der Elektronen, ausgenommen $\mathfrak{r}_{1}$ und $\mathfrak{r}_{2}$, integrieren, ferner über alle Spinkoordinaten summieren. Da das Störungsverfahren nicht konvergiert, wie bereits festgestellt wurde, soll nur qualitativ untersucht werden, wie der durch die Störung hervorgerufene Zusatz $\Delta w\left(\mathfrak{r}_{1}, \mathfrak{r}_{2}\right)$ zu dieser Wahrscheinlichkeit vom gegenseitigen Abstand der beiden Elektronen abhängt.

Aus (15) und (18) schließt man:

$$
\begin{aligned}
& \Delta w\left(\mathfrak{r}_{1}, \mathfrak{r}_{2}\right) \sim-\int \frac{d \mathfrak{g}}{h^{3}} \frac{4 \pi e^{2} \hbar^{2}}{g^{2}} \int \frac{2 d \mathfrak{p}_{1}^{\prime}}{h^{3}} \int \frac{2 d \mathfrak{p}_{2}{ }^{\prime}}{h^{3}} \\
& {\left[e^{\frac{i}{\hbar}\left(\mathfrak{p}_{1}^{\prime \prime} \mathfrak{r}_{1}+\mathfrak{p}_{2}^{\prime \prime} \mathrm{r}_{2}\right)}-e^{\frac{i}{\hbar}\left(\mathfrak{p}_{1}^{\prime \prime} \mathrm{r}_{2}+\mathfrak{p}_{2}^{\prime \prime} \mathfrak{r}_{1}\right)}\right]\left[e^{\frac{i}{\hbar}\left(\mathfrak{p}_{1}^{\prime} \mathfrak{r}_{1}+\mathfrak{p}_{2}^{\prime} \mathfrak{r}_{2}\right)}-e^{\frac{i}{\hbar}\left(\mathfrak{p}_{1}^{\prime} \mathfrak{r}_{2}+\mathfrak{p}_{2}^{\prime} \mathfrak{r}_{2}\right)}\right]} \\
& E_{\mathfrak{p}_{1}^{\prime} \mathfrak{p}_{2}{ }^{\prime} \mathrm{g}}-E_{0}^{\prime} \\
& \sim \int \frac{d \mathfrak{g}}{h^{3}} \frac{4 \pi e^{2} \hbar^{2}}{g^{2}} \int \frac{4 d \mathfrak{p}_{1} d \mathfrak{p}_{2}}{h^{6}} \frac{m}{\mathfrak{g}\left(\mathfrak{p}_{1}^{\prime}-\mathfrak{p}_{2}^{\prime}+\mathfrak{g}\right)}\left[e^{\frac{i}{\hbar} \mathfrak{g}\left(\mathfrak{r}_{1}-\mathbf{r}_{2}\right)}-e^{\frac{i}{\hbar}\left(\mathfrak{p}_{1}^{\prime}-\mathfrak{p}_{2}^{\prime}+\mathfrak{g}\right)\left(\mathfrak{r}_{1}-\mathfrak{r}_{2}\right)}\right] .
\end{aligned}
$$

Für weite Abstände der beiden Teilchen (d.h. $\left|\mathfrak{r}_{1}{ }^{\star} \mathfrak{r}_{2}\right| \gg \hbar / P$ ) rührt der Hauptbeitrag zu diesem Integral von dem ersten Gligd $e^{\frac{i}{\hbar} \mathfrak{g}\left(\mathfrak{r}_{1}-\mathfrak{r}_{\Perp}\right)}$ und von kleinen Werten von $g$ her. Man entnimmt 
aus (14), daß für kleine Werte von $g$ das Integral (19) wie

$$
\Delta w_{\left|\mathbf{r}_{1}-\mathbf{r}_{2}\right|>\hbar / P} \sim-\int \frac{d \mathfrak{g}}{g} e^{\frac{i}{\hbar} \mathfrak{g}\left(\mathbf{r}_{1}-\mathbf{r}_{2}\right)}
$$

variiert. Also folgt:

$$
\Delta w_{\left|\mathfrak{r}_{1}-\mathfrak{r}_{2}\right| \gg \hbar / P} \sim-\mathrm{const} /\left|\mathfrak{r}_{1}-\mathfrak{r}_{2}\right|^{2} .
$$

Die Coulomb-Kräfte haben also die Tendenz, auch über weite Abstände eine Annäherung der Elektronen unwạhrscheinlicher zu machen. Diese Tendenz wird sich zwar kaum bis zu beliebig großen Abständen fortsetzen, da eben die Divergenz des Störungsverfahrens bei kleinen $g$ Werten zeigt, daß dort schließlich etwas anderes geschieht. Aber es liegt nahe, anzunehmen, daß die vom Coulomb-Feld möglicherweise hervorgerufene Ordnung der Elektronen durch $\mathrm{Ab}$ weichungen des Typus (21) von der üblichen Wahrscheinlichkeitsverteilung im Fermi-Gas charakterisiert ist.

2. Die Energie des geordneten Zustandes

a) Jede Lokalisierung eines Elektrons bringt vermöge der Unbestimmtheitsrelation eine kinetische Energie mit sich. Diese Beziehung ist für die Elektronen eines Fermi-Gases etwas anders als für ein einzelnes freies Elektron, da zum Aufbauen eines Wellenpakets nur unbesetzte $\mathrm{Zu}$ stände verwendet werden können. Um diese Be- ziehung zu ermitteln, fragen wir zuerst, welche Art von Wellenpaketen aus den Zuständen aufgebaut werden können, die außerhalb der Kugel vom Radius $P$ im Impulsraum, aber in der unmittelbaren Nähe ihrer Oberfläche liegen; für ein solches Wellenpaket ist dann die kinetische Energie zu berechnen. Da es sich hier nur um qualitative Betrachtungen handelt, genügt es wohl, irgendeine plausible Form für das Wellenpaket zu wählen. Wir werden also etwa voraussetzen, daß erstens die Amplitude im Impulsraum im Inneren der Kugel vom Radius $P$ verschwindet und mit dem Abstand von der Oberfläche der Kugel exponentiell abfällt; daß sie zweitens ihren größten Wert an einem bestimmten Punkt der Oberfläche hat und auch mit dem Abstand von diesem Punkt exponentiell abfällt. Wenn $\zeta$ den Cosinus des Winkels zwischen dieser ausgezeichneten Impulsrichtung und dem Impuls bedeutet, so soll also die Amplitude im Impulsraum etwa die Form

$$
e^{a(\zeta-1)-\beta(p-P)}
$$

haben. Das zugehörige Wellenpaket im Koardinatenraum hat dann die Form

$$
f(\mathfrak{r})=\int d \mathfrak{p} e^{a(\zeta-1)-\beta(p-P)+\frac{i}{\hbar} \mathfrak{p} \mathfrak{r}} .
$$

Führt man im Impulsraum die Polarkoordinaten $p, \zeta, \varphi$ ein und bezeichnet den Cosinus des Winkels zwischen $\mathfrak{r}$ und der ausgezeichneten Impulsrichtung (die zugleich die Polarachse ist) mit $\eta$, so geht (23) über in

$$
f(\mathfrak{r})=\int p^{2} d p d \zeta d \varphi e^{\frac{i}{\hbar} p r\left(\zeta \eta+\sqrt{\left(1-\zeta^{2}\right)\left(1-\eta^{2}\right)} \cos \varphi\right)+\alpha(\zeta-1)-\beta(p-P)} .
$$

Für die Auswertung setzten wir voraus, daß $\beta P \gg 1$ ist, d.h. daß das Wellenpaket sich nur aus Impulsen in der unmittelbaren Umgebung der Kugeloberfläche zusammensetzt. Ferner soll $f(\mathfrak{r})$ für die Fälle $\alpha \gg 1$ und $\alpha=0$ gesondert berechnet werden.

1. $\alpha \gg 1$. Hier setzt man zweckmäßig $\zeta=1-\epsilon^{2} / 2 \quad(\epsilon \ll 1)$ und erhält, sofern $\eta \gg 1 / \sqrt{a}$ ist:

$$
\begin{aligned}
f(\mathfrak{r}) & \approx \int_{P}^{\infty} p^{2} d p \int \varepsilon d \varepsilon d \varphi e^{\frac{i}{\hbar} p r\left(\eta-\frac{\varepsilon^{2}}{2} \eta+\varepsilon \sqrt{1-\eta^{2}} \cos \varphi\right)-a \frac{\varepsilon^{2}}{2}-\beta(p-P)} \\
& \approx \frac{P^{2}}{\beta-\frac{i}{\hbar} r \eta} \int \varepsilon d \varepsilon d \varphi e^{\frac{i}{\hbar} \operatorname{Pr}\left(\eta-\frac{\varepsilon^{2}}{2} \eta+\varepsilon \sqrt{1-\eta^{2}} \cos \varphi\right)-a \frac{\varepsilon^{2}}{2}} \\
& \approx \frac{2 \pi P^{2}}{\left(\beta-\frac{i}{\hbar} r \eta\right)\left(a+\frac{i}{\hbar} \operatorname{Pr} \eta\right)} e^{\frac{i}{\hbar} \operatorname{Pr} \eta-\frac{\left(\frac{P r}{\hbar}\right)^{2}\left(1-\eta^{2}\right)}{2\left(a+\frac{i}{\hbar} \operatorname{Pr} \eta\right)}}
\end{aligned}
$$


also

$$
|f(\mathfrak{r})|^{2} \approx \frac{4 \pi^{2} P^{4}}{\left[\beta^{2}+(r \eta / \hbar)^{2}\right]\left[\alpha^{2}+(\operatorname{Pr} \eta / \hbar)^{2}\right]} e^{-\frac{\alpha(\operatorname{Pr} / \hbar)^{2}\left(1-\eta^{2}\right)}{a^{2}+(\operatorname{Pr} \eta / \hbar)^{2}}} .
$$

2. $\alpha=0$. Hier führt man als Polarachse im $p$ Raum am besten die Richtung von $\mathfrak{r}$ ein und erhält:

$$
\begin{aligned}
f(\mathfrak{x}) & =2 \pi \int_{P}^{\infty} p^{2} d p \int d \zeta e^{\frac{i}{\hbar} p r \zeta-\beta(p-P)} \\
& \approx 4 \pi P \frac{\cos \frac{P r}{\hbar}+\frac{\beta \hbar}{r} \sin \frac{P r}{\hbar}}{\beta^{2}+\frac{r^{2}}{\hbar^{2}}} .
\end{aligned}
$$

$\mathrm{Zu}$ diesen Ausdrücken kommen noch Normierungsfaktoren, wenn man fordert, daß etwa im Wellenpaket genau ein Elektron enthalten sein soll. Diesen Normierungsfaktor bestimmt man am besten, indem man zuerst den Normierungsfaktor im Impulsraum aufsucht. Aus (22) erhält man ohne Schwierigkeit für den Normierungsfaktor im Impulsraum

$$
\sqrt{2 a \beta / \pi P^{2}\left(1-e^{-4 a}\right)} .
$$

Dementsprechend ist $f(\mathfrak{r})$ in (25) und (27) mit

$$
\sqrt{2 \alpha \beta / \pi \mathrm{P}^{2} h^{3}\left(1-e^{-4 a}\right)}
$$

zu multiplizieren, wenn $|f(\mathfrak{x})|^{2}$ die Wahrscheinlichkeitsverteilung für ein einzelnes Elektron bedeuten soll.

Die mit dem Wellenpaket verbundene kinetische Energie erhält man aus der Wahrscheinlichkeitsverteilung im Impulsraum. Dabei interessiert uns der Überschuß der kinetischen Energie über den Minimalwert $P^{2} / 2 \mathrm{~m}$, den das Elektron hätte, wenn es direkt zur Oberfläche der Impulskugel gehören würde. Der Überschuß, der zur Wellenfunktion (22) gehört, ergibt sich zu

$$
\Delta E_{\mathrm{kin}}=P / 2 m \beta ;
$$

er ist von der Konstanten $\alpha$ unabhängig.

b) Nachdem durch (30) festgestellt ist, wieviel kinetische Energie notwendig ist, damit eine Lokalisierung eines Elektrons in Wellenpaketen der Art (26) und (27) ermöglicht wird, muß jetzt die potentielle Energie berechnet werden, die man gewinnen kann, wenn man die Elektronen mit Hilfe solcher Wellenpakete ordnet.
Die energetisch günstigste Ordnung scheint etwa von folgender Art zu sein: Wir betrachten den Ort eines bestimmten Elektrons und nehmen an, daß relativ zu diesem Elektron die anderen - ähnlich wie im Kronigschen Entwurf* - in einer Art von Kristallgitter geordnet sind in der Weise, daß sie in Wellenpaketen der Form (26) und (27) lokalisiert sind, deren Mittelpunkte in einer regelmäßigen Anordnung um das betreffende Elektron herum liegen; man könnte etwa an ein Gitter vom CsCl-Typ denken, bei dem die Elektronen verschiedener Spinrichtung den beiden Ionensorten entsprechen. Dabei wird der Gitterabstand erheblich kleiner sein als die Größe $\beta \hbar$, die man etwa als „Radius“ des Wellenpakets definieren könnte (wie sich durch eine genauere Berechnung der höchsten bei dieser Form des Wellenpakets erreichbaren Dichte ergibt), andererseits natürlich (im Gegensatz zu K r o nig) viel größer als der Gitterabstand im Metallgitter, mit dem das Elektronengitter nichts zu tun hat.

Diese Ordnung ist nicht identisch mit einer Ordnung, bei der man etwa jedes Elektron dieser geordneten Phase (das wird jedoch nur ein kleiner Bruchteil aller Leitungselektronen sein) im Raum in einem Wellenpaket an einem Gitterpunkt lokalisiert. Denn bei der letztgenannten Ordnung ist die Wahrscheinlichkeit, daß zwei Elektronen einander nahe kommen, größer als bei der erstgenannten. Man könnte aber vielleicht mit der letztgenannten Ordnung als „0-ter" Näherung beginnen und dann mit Hilfe einer Störungsrechnung zu einer Wellenfunktion kommen, die im wesentlichen die erstgenannte Ordnung repräsentiert.

Die erstgenannte Ordnung, um die es sich im folgenden stets handeln soll, ist auf Grund der Coulombschen Abstoßung der Elektronen energetisch günstiger als die gleichmäßige Verteilung der Elektronen im Fermi-Gas. Zwar sorgt auch im Fermi-Gas das Pauli-Prinzip dafür, daß zwei Elektronen mit gleicher Spinrichtung einander nicht zu nahe kommen kốnnen, und dieser Effekt führt zu einer Verringerung der Aufenthaltswahrscheinlichkeit eines Elektrons in einem Abstand von der Ordnung $\hbar / P$ von einem anderen Elektron gleicher Spinrichtung. Aber die genannte 
Ordnung verringert die Aufenthaltswahrscheinlichkeit auch bei Elektronen verschiedener Spinrichtung. Sieht man zunächst von der Wirkung der Fermi-Statistik ab, so kann man die Cou$10 \mathrm{mb}$-Energie in folgender Weise abschätzen. Wenn jeder Gitterpunkt mit einem Wellenpaket besetzt wäre, so erhielte man eine einigermaßen gleichmäßig verteilte elektrische Ladungsdichte, die durch die Ladungen der Ionen im Mittel zu Null kompensiert wird. $\mathrm{Zu}$ dieser Ladungsdichte fehlt aber in Wirklichkeit das Wellenpaket an der Stelle des Elektrons, um dessen Umgebung es sich handelt. Die Dichte dieses Wellenpakets fällt nach (26) in einem Gebiet zwischen Abständen der Ordnung $\frac{\hbar}{P} \sqrt{a}$ und $\hbar \beta$ wie $1 / r^{2}$ ab, verhält sich dort also, wie es nach (21) zu erwarten ist.

Den Gewinn an potentieller Energie kann man daher erhalten, indem man die elektrostatische Wechselwirkung zwischen einem Wellenpaket der Form (26), (27) und einem in seinem Mittelpunkt gelegenen Elektron berechnet. So erhält man für den Gewinn an Coulomb-Energie pro Teilchen unter Berücksichtigung von (26) und (29)

$$
\begin{aligned}
& \Delta E_{\text {Coul }}= \\
& \frac{2 \alpha \beta}{\pi P^{2}\left(1-e^{-4 a}\right) h^{3}} \int_{0}^{\infty} \frac{e^{2}}{r}|f(r)|^{2} 4 \pi r^{2} d r .
\end{aligned}
$$

Die Auswertung ergibt:

1. für $\alpha \gg 1$ :

$\Delta E_{\mathrm{Coul}} \approx \frac{2 e^{2} a}{h \beta\left(1-e^{-4 a}\right)}\left[\lg \frac{\beta^{2} P^{2}}{a}+1,56\right]$,

2. für $\alpha=0$ :

$$
\Delta E_{\mathrm{Coul}} \approx \frac{4 e^{2}}{h \beta} \lg (2 P \beta) .
$$

Der charakteristische logarithmische Faktor in diesen beiden Ausdrücken rührt von dem Integral $\int 4 \pi r^{2} d r \cdot e^{2} / r \cdot 1 / r^{2}$ her, das über das Gebiet zu erstrecken ist, in dem das Wellenpaket wie $1 / r^{2}$ abfällt. Man kann die Formeln (32) und (33) quali- tativ durch die Annahme deuten, daß die obere Grenze dieses Gebiets durch $r=\beta \hbar$, die untere durch $\frac{\hbar}{P} \sqrt{a}$ bzw. (für $\alpha=0$ ) durch $\hbar / P$ gegeben ist.

Bei dieser Rechnung wurde aber bisher nicht berücksichtigt, daß die Elektronen der FermiStatistik genügen. Da diese schon von selbst Einflüsse der Art (21) hervorbringt, wird der Faktor vor dem logarithmischen Glied von der FermiStatistik verkleinert werden. Diese Frage bedarf einer etwas ausführlicheren Untersuchung:

Wir betrachten etwa ein gewöhnliches FermiGas und teilen alle Elektronen in zwei Gruppen ein: solche, deren Impulse kleiner sind als $P-\Delta P$, und die Elektronen der „Oberflächenschicht“ (im Impulsraum), deren Impulse zwischen $P-\Delta P$ und $P$ liegen. Die Wahrscheinlichkeit, ein Elektron an der Stelle $\mathfrak{r}_{1}$ und ein zweites an der Stelle $\mathfrak{r}_{2}$ zu finden, ist in der Fermi-Statistik ganz allgemein gegeben durch den Ausdruck

$$
\begin{aligned}
& w\left(\mathfrak{r}_{1}, \mathfrak{r}_{2}\right)= \\
& \frac{1}{N(N-1)}\left[n\left(\mathfrak{r}_{1}\right) n\left(\mathfrak{r}_{2}\right)-\frac{1}{2}\left|n^{2}\left(\mathfrak{r}_{1}, \mathfrak{r}_{2}\right)\right|\right],
\end{aligned}
$$

wobei $N$ die Anzahl aller Elektronen, $n(\mathfrak{r})$ die räumliche Dichte (2) und $n\left(\mathfrak{r}_{1}, \mathfrak{r}_{2}\right)$ die von Dir a c eingeführte gemischte Dichte bedeutet:

$$
n\left(\mathfrak{r}_{1}, \mathfrak{r}_{2}\right)=\frac{2}{h^{3}} \int_{0}^{P} d \mathfrak{p} e^{\frac{i}{\hbar} \mathfrak{p}\left(\mathfrak{r}_{1}-\mathfrak{r}_{2}\right)} .
$$

Diesen Ausdruck $w\left(\mathfrak{r}_{1}, \mathfrak{r}_{2}\right)$ teilen wir in drei Anteile: Der erste [die ersten beiden Zeilen in (36)] soll von der Hauptmenge der Elektronen $(|\mathfrak{p}|<P$ $-\triangle P$ ) herrühren, der zweite [dritte Zeile in (36)] von den Elektronen der Oberfläche $(P-\Delta P<|\mathfrak{p}|$ $<P$ ), und der dritte [die letzten beiden Zeilen in (36)] von den gemischten Gliedern. Zur Vereinfachung der Rechnung setzen wir $\left|\mathfrak{r}_{1}-\mathfrak{r}_{2}\right|=s$, nehmen $\Delta P \ll P$ an und vernachlässigen alle Glieder mit $\Delta P^{3}, \Delta P^{4}$ usw.; ferner sei $\hbar / \Delta P \gg s \gg \hbar / P$, und wir berücksichtigen nur die jeweils höchsten Potenzen von $s P / \hbar$. Dann ergibt sich:

$$
\begin{aligned}
N(N-1) w\left(\mathrm{r}_{1}, \mathfrak{r}_{2}\right) \approx\left(\frac{8 \pi}{h^{3}}\right)^{2}\left(P^{6}-6 P^{5} \Delta P+15 P^{4} \Delta P^{2}\right) & \\
& -\frac{1}{2 \pi^{4}}\left(\frac{P^{2} \cos ^{2}\left(\frac{P s}{\hbar}\right)}{\hbar^{2} s^{4}}+\frac{P^{2} \Delta P \sin \frac{2 P s}{\hbar}}{\hbar^{3} s^{3}}-\frac{\left.P^{2} \Delta P^{2} \cos \frac{2 P s}{\hbar}\right)+}{\hbar^{4} s^{2}}\right)
\end{aligned}
$$




$$
\begin{aligned}
& +\left(\frac{8 \pi}{h^{3}}\right)^{2} 9 P^{4} \Delta P^{2}-\frac{1}{2 \pi^{4}} \frac{P^{2} \Delta P^{2} \sin ^{2} \frac{P s}{\hbar}}{\hbar^{4} s^{2}} \\
& +\left(\frac{8 \pi}{h^{3}}\right)^{2}\left(6 P^{5} \Delta P-24 P^{4} \Delta P^{2}\right) \\
& +\frac{1}{2 \pi^{4}}\left(\frac{P^{2} \Delta P \sin \frac{2 P s}{\hbar}}{\hbar^{3} s^{3}}+\frac{P^{2} \Delta P^{2}}{\hbar^{4} s^{2}}\left(2 \sin ^{2} \frac{P s}{\hbar}-\cos ^{2} \frac{P s}{\hbar}\right)\right) .
\end{aligned}
$$

Beim Studium von (36) erkennt man zunächst, daß die Summe über alle fünf Zeilen rechts nur ein Glied der Form

$$
-\frac{P^{2}}{2 \pi^{4}} \frac{\cos ^{2}(P s / \hbar)}{\hbar^{2} s^{4}}
$$

ergibt. Die Abweichung der Gesamt-Wahrscheinlichkeit von der gleichmäßigen Verteilung fällt also sehr schnell mit $s$ ab (wie $s^{-4}$ ) und führt daher nicht zu einem logarithmischen Glied in der Cou lo mb-Energie. Betrachtet man aber nur die Elektronen der Oberfläche, d.h. die dritte Zeile in (36), so enthält die zugehörige Wahrscheinlichkeit ein Glied

$$
\frac{P^{2} \Delta P^{2}}{2 \pi^{4} \hbar^{4} s^{2}} \sin ^{2} \frac{P s}{\hbar},
$$

das wie $s^{-2}$ abfällt und daher ein logarithmisches Glied in der Coulomb-Energie hervorruft von derselben Form wie Gln. (31) bis (33). Dieses Glied wird in der Gesamtbilanz aber gerade kompensiert durch ein Glied in der fünften Zeile, das von dem Austausch zwischen den Oberflächenelektronen und den übrigen Elektronen herrührt.

Im ganzen kann man also feststellen: Allein die Tatsache, daß in der Fermi-Statistik zwei Elektronen mit gleicher Spinrichtung nicht am gleichen Ort sein können, bewirkt bereits, daß für die Elektronen der Oberfläche die Wahrscheinlichkeit die Form

$$
w\left(\mathfrak{r}_{1} \mathfrak{r}_{2}\right) \sim a-\frac{b}{\left|\mathfrak{r}_{1}-\mathfrak{r}_{2}\right|^{2}}
$$

hat, und zwar gilt dies im Bereich $\hbar / P \ll\left|\mathrm{x}_{1}-\mathrm{r}_{2}\right|$ $\ll \hbar / \Delta P$. Dafür wird aber die Wahrscheinlichkeit, in der Nähe eines Oberflächenelektrons ein inneres zu finden, etwas erhöht durch ein Glied der Form

$$
w\left(\mathfrak{r}_{1} \mathfrak{r}_{2}\right) \sim a^{\prime}+\frac{b^{\prime} \Delta P}{\left|\mathfrak{r}_{1}{ }^{0}-\mathfrak{r}_{2}{ }^{i}\right|^{2}},
$$

das gerade ausreicht, um im ganzen die Anderung der Ladungsverteilung in der Umgebung des Ober- flächenelektrons so weit zu kompensièren, daß im gewöhnlichen Fermi-Gas kein logarithmisches Glied der Form (31) auftritt. Dabei ist noch zu beachten, daß die ganzen Änderungen (36) in der Wahrscheinlichkeitsverteilung von den Elektronen gleicher Spinrichtung herrühren; die Wahrscheinlichkeit, ein Elektron entgegengesetzter Spinrichtung in der Nähe eines Elektrons zu finden, ist völlig gleichmäßig verteilt.

Es fragt sich nun, wie die Funktion $w\left(\mathfrak{r}_{1} \mathfrak{r}_{2}\right)$ verändert wird, wenn man die Oberflächenschicht des Fermi-Gases dadurch modifiziert, daß man Wellenpakete der Form (23) an die Stelle der ebenen Wellen treten läßt und die Wellenpakete in der beschriebenen Weise ordnet. Zunächst wird nach den vorhergehenden Utberlegungen die Einführung der Wellenpakete nur auf die Wahrscheinlichkeitsverteilung der Elektronen entgegengesetzten Spins einen erheblichen Einfluß ausüben; die Coulomb-Energie (31) wird bei Berücksichtigung der Fermi-Statistik also im Mittel zunächst etwa halb so groß sein wie bei klassischer Statistik. Andererseits wird das Glied (38), das entgegengesetztes Vorzeichen hat, durch die Einführung der Wellenpakete erhöht werden, und damit wird das logarithmische Glied in der Cou$10 \mathrm{mb}$-Energie weiter verkleinert. Es wäre sogar möglich, daß diese Erhöhung des Gliedes (38) den Energiegewinn (31) überkompensiert, so daß kein Energiegewinn von der Form des logarithmischen Gliedes übrig bliebe. Dies möchten wir aber nicht annehmen, da es möglich sein dürfte, die Wellenfunktion nur hinsichtlich der relativen Lage der Elektronen entgegengesetzten Spins relativ zum gewöhnlichen F e r mi-Gas erheblich abzuändern. Wir glauben daher, daß das logarithmische Glied (31) noch um einen Zahlfaktor, den wir $z$ nennen wollen, verkleinert werden muß - wobei $z$ sicher größer als 2 sein wird -, daß es aber sein Vorzeichen beibehält. Die Tatsache, daß man durch die Ordnung in Wellenpaketen nur bei der C o u l o mb- 
Energie der Elektronen entgegengesetzten Spins einen größeren Betrag gewinnen kann, hat auch noch die interessante Folge, daß ferromagnetische Metalle nicht supraleitend werden sollten; denn bei ihnen ist die Spinrichtung aller Elektronen bei tiefen Temperaturen gleich. Tatsächlich ist bisher kein ferromagnetischer Supraleiter bekannt.

Die nächste Frage ist, welchen Weŕt für $r$ man als untere Grenze des Integrals $d r / r$ in (31) einzusetzen hat. In (33) sowohl wie im Ausdruck (37) für die Oberflächenschicht des F e r m i-Gases ist die untere Grenze etwa durch $r \sim \hbar / P$ gegeben. Andererseits ist die untere Grenze in (32) $\frac{\hbar}{P} \sqrt{\alpha}$, und es könnte sich außerdem bei der genaueren Untersuchung der Wellenfunktion im Konfigurationsraum ergeben, daß eine Wahrscheinlichkeitsverteilung von der Art (37) sich nur bis herunter zu Radien von der Größenordnung der Abstände der Elektronen $\left(\sim \Delta n^{-1 / 3}\right)$ ohne erheblichen Aufwand an kinetischer Energie fortsetzen läßt. Für die mehr qualitativen Schlüsse der folgenden Abschnitte macht es keinen allzu großen Unterschied, welchen der drei genannten Radien man unter dem Logarithmus einsetzt, und wir werden daher willkürlich als untere Grenze $r \sim \hbar / P$ benutzen.

Die Fermi-Statistik bewirkt auch, daß die Dichte $\Delta n$ der Supraleitungselektronen bei gegebenem $\beta$ einen gewissen Wert nicht übersteigen kann. Da das Wellenpaket im Impulsraum nach (28) ein Volumen der Größenordnung $\pi P^{2}$ $\left(1-e^{-4 a}\right) / 2 \alpha \beta$ erfüllt, wird man in der üblichen Weise für die maximale Dichte

$$
\Delta n \sim \pi P^{2}\left(1-e^{-4 a}\right) / \alpha \beta h^{3}
$$

setzen können; die Dichte im Phasenraum übersteigt dann nirgends den Wert $2 / h^{3}$, wie es nach dem Pauli-Prinzip sein muß. So erhält man schließlich für die gesamte Energiedichte der geordneten Phase:

$$
\begin{aligned}
& u \approx \Delta n\left[\frac{P}{2 m \beta}-\frac{2 e^{2}}{\pi \beta \hbar} \lg (2 \beta P)\right]^{\circ} \\
& \approx \frac{a h^{3}}{2 \pi\left(1-e^{-4 a}\right) P m} \\
& \Delta n^{2}\left[1-\frac{1}{Z} \lg \frac{2 \pi P^{3}\left(1-e^{-4 a}\right)}{a h^{3} \Delta n}\right]
\end{aligned}
$$$$
\text { wobei } \quad Z=\frac{z \pi \hbar P}{4 e^{2} m}=\frac{z \pi}{4} \frac{\hbar v}{e^{2}}
$$

eine reine $\mathrm{Z}$ ahl ist; $v=P / m$ bedeutet die Geschwindigkeit der Elektronen. $\hbar v / e^{2}$ wäre im Wasserstoffatom genau gleich 1 ; hier kann $\hbar v / e^{2}$ größer oder kleiner als 1 sein, je nach dem Wert der effektiven Masse der Elektronen in dem betreffenden Energieband. Der Faktor $z \pi / 4$ wird jedenfalls größer als 1 sein, sein Wert könnte aber nur durch eine sehr viel genauere Rechnung ermittelt werden. In (40) ist ferner nicht berücksichtigt, daß die $\Delta n$ Elektronen auch in der ungeordneten Phase eine gewisse kinetische Energie über $P^{2} / 2 m$ hinaus hätten, die in der Energiedifferenz zwischen der geordneten und der ungeordneten Phase zu subtrahieren wäre. Da eine ähnliche, durch das Pa uli Prinzip bedingte Energie aber vielleicht auch bei den Wellenpaketen auftritt, und da sie sicher kleiner ist als das erste Glied in (40), haben wir sie in dieser groben Abschätzung weggelassen.

c) Bevor die Gln. (40) und (41) auf das gestellte physikalische Problem angewendet werden, muß der Genauigkeitsgrad der ihr zugrunde liegenden Abschätzung diskutiert werden. Zunächst folgt schon aus der Art, wie hier die Unbestimmtheitsrelation benützt worden ist, daß Faktoren von der Größenordnung 1 in allen Gliedern der Formel zugelassen werden müssen. Darüber hinausgehend kann die Ableitung der Gln. (40) und (41) nur im Sinne eines Ritzschen Verfahrens verstanden werden; es ist ja nicht gezeigt worden, daß sich eine Wellenfunktion des Gesamtsystems, die der beschriebenen Ordnung entspricht, wirklich einstellt, sondern es kann nur gezeigt werden, daß der so geordnete Zustand energetisch günstiger ist als der normale Zustand des Fermi-Gases. Ferner ist nicht einmal gezeigt worden, daß es eine Wellenfunktion gibt, die zu der postulierten Wahrscheinlichkeitsverteilung im Konfigurationsraum führt. Allerdings würde die Aufstellung einer solchen Wellenfunktion grundsätzlich Betrachtungen über den vieldimensionalen Konfigurationsraum erfordern und damit $\mathrm{zu}$ erheblichen mathematischen Schwierigkeiten führen.

Wellenfunktionen für die Elektronen im Metall, die auch die Wechselwirkung der Elektronen berücksichtigen sollen, sind schon früher von Wig ner und Seit ${ }^{6}$ aufgestellt und untersucht worden. Die Wellenfunktionen, an die wir bei der Theorie der Supraleitung denken, könnten gegenüber dem Ansatz von Wigner und Seitz etwa in

\footnotetext{
6 E. Wigner u. F. Seitz, Physic. Rev. 46, 509
} [1934]; E. Wi gner, Physic. Rev. 46, 1002 [1934]. 
folgender Weise beschrieben werden: Ähnlich wie dort sollte die Wellenfunktion ein Produkt von zwei Determinanten sein, wobei jede Determinante sich auf die Elektronen einer Spinrichtung bezieht. In jeder Determinante sollen, im Gegensatz zu Wigner und Seitz, als Elektronenzustände einfach die üblichen ebenen Wellen der Dir acschen "See“ stehen, nur die ebenen Wellen der Oberfläche der "See“ sind durch Wellenpakete zu ersetzen, deren Mittelpunkte den Cs- bzw. Cl-Kernen eines CsCl-Gitters großen Gitterabstandes (10 ̊ und mehr!) entsprechen. Außerdem müßte wohl das Produkt der beiden Determinanten noch multipliziert werden mit einem Faktor der Art

$$
\stackrel{{ }^{k}+k}{\Pi}-\left(1-\frac{a}{b^{2}+r^{2}{ }_{k_{+}, k}}\right)
$$

(die Indices $k_{+}$bzw. $k_{-}$beziehen sich auf die Elektronen positiver bzw. negativer Spinrichtung an der Oberfläche der „See“), der dafür sorgt, daß die Elektronen entgegengesetzter Spinrichtung einander nicht zu nahe kommen. Dies wäre ein möglicher Ansatz für eine solche Wellenfunktion; die Auswertung würde zu großen mathematischen Schwierigkeiten führen.

Wenn man mit diesen Vorbehalten die Gl. (40) diskutiert, so wird man schließen, daß sich am absoluten Nullpunkt die Dichte $\Delta n$ einstellen wird, die den Ausdruck (40) zum Minimum macht. Dabei kann man zunächst $\alpha$ als gegeben betrachten, und später auch $\alpha$ variieren und $u$ als Funktion von $\alpha$ zum Minimum machen. Durch Differenzieren findet man aus (40):

$$
\Delta n_{\min }=\frac{2 \pi P^{3}}{h^{3}} \frac{1-e^{-4 a}}{a} e^{-Z-1 / 2}
$$

und

$$
u_{\min }=\frac{2 \pi P^{5}}{m h^{3}} \frac{1-e^{-4 a}}{a} \frac{e^{-2 Z-1}}{2 Z} .
$$

Wenn die hier diskutierte Ordnung eine Beschreibung der supraleitenden Phase sein soll, so muß die eben berechnete Energiedichte in der Größenoŕrdnung übereinstimmen mit der durch die Temperaturbewegung verursachten mittleren Energiedichte der Leitungselektronen in der Nähe des Sprungpunktes; sie sollte also etwa um den Faktor $\left(k T_{0} / E\right)^{2}$ kleiner sein als die mittlere kinetische Energiedichte des Fermi-Gases, wobei $T_{0}$ die Temperatur des Sprungpunktes und $E=P^{2} / 2 m$ die kinetische Energie eines Elektrons an der Oberfläche der Impulskugel bedeutet. Wenn man für normale Supraleiter $k T_{0} / E \sim 10^{-4} \mathrm{ab}-$ schätzt, so folgt daraus in grober Näherung

$$
e^{-2 Z-1} \sim 10^{-8} \text {, d. h. } Z \sim 8,7 \text {. }
$$

Ein solcher Zahlwert für $Z$ ist zwar höher, als man nach der Abschätzung (41) zunächst erwarten würde, aber auch nicht unplausibel hoch. Die merkwürdig niedrigen Sprungtemperaturen bei der Supraleitung würden also, wenn die hier versuchte Deutung richtig ist, indirekt eine Folge der logarithmischen Abhängigkeit der Energiedichte $u$ von der Ladungsdichte $\Delta n$ sein. In der Tat ist eine Deutung dieser Art wohl die einzige Möglichkeit, zu den gemessenen niedrigen Sprungtemperaturen zu kommen, wenn man die großen Coulombschen Kräfte für die Ordnung der Phase verantwortlich macht. Denn die elektrostatischen Kräfte würden sonst immer zu Temperaturen mindestens von der Ordnung der CurieTemperaturen in Ferromagneten führen. Man hat deshalb gelegentlich versucht, die magnetischen Kräfte für die Supraleitung verantwortlich $\mathrm{zu}$ machen. Aber bisher ist keine Wirkung der magnetischen Kräfte bekannt, die gegen die viel stärkere Wirkung der übrigen Kräfte einen geordneten Zustand herbeiführen könnte.

Vielleichtkann man aber zwei empirische Argumente für die hier versuchte Deutung anführen. Wenn (43) richtig ist, so hängt $T_{0}$ sehr empfindlich von $Z$ und damit von der Besetzung des Energiebandes und der effektiven Masse ab. Man sollte also bei verschiedenen Substanzen sehr große Schwankungen von $T_{0}$ beobachten; tatsächlich scheinen die Sprungtemperaturen verschiedener Stoffe bis zu zwei Zehnerpotenzen auseinander $\mathrm{zu}$ liegen (z. B. Hf $0,3^{\circ} \mathrm{K}, \mathrm{Zn} 0,7^{\circ} \mathrm{K}$, $\mathrm{NbN} 20,4^{\circ} \mathrm{K}$ ), während die $\mathrm{Cu}$ rie-Temperaturen bei den meisten ferromagnetischen Stoffen kaum um einen Faktor 4 auseinanderliegen (sofern man von der besonderen Gruppe von Stoffen absieht, bei denen die Magnetisierung nicht durch die Austauschkraft, sondern durch magnetische Wirkungen zustandekommt).

Zweitens sollte man bei der Gültigkeit von (43) erwarten, daß die Sprungtemperatur um so höher liegt, je kleiner $Z$, d. h. je größer die effektive Masse und je kleiner die Geschwindigkeit der Elektronen ist. Ein Anzeichen für einen derartigen Zusammenhang kann man in der Tatsache 
erblicken, daß Verbindungen wie $\mathrm{ZrN}, \mathrm{NbH}, \mathrm{NbN}$, MoC einen höheren Sprungpunkt haben als die betreffenden reinen Elemente.

d) Der mittlere Impuls, der zu einem Wellenpaket der Form (22) gehört, beträgt

$$
\bar{p}=P(\widetilde{\operatorname{tg} 2} \alpha-1 / 2 \alpha) \text {. }
$$

Die Stromdichte, die von den Elektronen in der geordneten Phase herrührt, wird dementsprechend

$$
s=\Delta n e \frac{P}{m}(\operatorname{stg} 2 \alpha-1 / 2 \alpha) .
$$

Gl. (43) zeigt, daß für $\alpha$ der energetisch günstigste Wert $\alpha=0$ ist; der stabilste Zustand ist also auch hier der Zustand ohne Strom. Dies bedeutet aber noch nicht, daß sich bei tiefen Temperaturen immer dieser Zustand ohne Strom einstellt. Denn jedenfalls liegt die geordnete Phase auch bei anderen Werten von $\alpha$ energetisch tiefer als die ungeordnete. Es hängt zunächst also von dem $\alpha$-Wert des ersten „Kristallkeims“ ab, welchen Strom die geordnete Phase erhält. Die Zusammenstöße mit dem Ionengitter werden dann zwar gelegentlich Elektronen aus der geordneten Phase entfernen und wieder andere Elektronen in diese Phase hereinbringen. Da aber neue Elektronen nur in Wellenpaketen mit dem gleichen $\alpha$ Wert gebunden werden können, besteht kaum Aussicht, daß sich der Strom der geordneten Phase durch Zusammenstöße mit dem Ionengitter merklich ändern könnte, sobald einmal viele Elektronen in die geordnete Phase übergegangen sind. Wie die späteren Rechnungen zeigen, ist es auch denkbar, daß bei von Null verschiedenen Temperaturen unter dem Sprungpunkt ein von Null verschiedener $\alpha$-Wert thermodynamisch stabil ist, obwohl der Zustand tiefster Energie $\alpha=0$ ist. Diese thermodynamischen Fragen bedürfen noch einer ausführlichen Untersuchung.

\section{Thermodynamische Fragen}

Bei von Null verschiedenen Temperaturen hängt die Energie, die man gewinnt, wenn man Elektronen aus der ungeordneten in die geordnete Phase überführt, auch von der Temperatur ab. Denn selbst wenn die geordnete Phase praktisch keine angeregten Zustände' besitzt, so daß von ihrer spezifischen Wärme ganz abgesehen werden kann, so hat doch die ungeordnete Phase eine spezifische Wärme. Die mittlere Temperaturenergie der un- geordneten Phase wird nun in zweierlei Weise durch die Überführung von Elektronen in die geordnete Phase verändert; erstens nimmt diese Energie ab, weil die Anzahl der Elektronen in der ungeordneten Phase geringer wird, und zweitens werden den Elektronen in der ungeordneten Phase angeregte Zustände durch die geordnete Phase weggenommen, da diese Zustände dann besetzt sind. An den Stellen der Oberfläche der Impulskugel, die von den Wellenpaketen des geordneten Zustandes bedeckt sind, werden die angeregten Zustände, die sonst für die spezifische Wärme maßgebend sind, ganz oder zum Teil besetzt sein, die spezifische Wärme wird also um einen von dieser bedeckten Oberfläche und von $\Delta n$ abhängigen Wert verkleinert.

Leider ist es wohl ganz unmöglich, diesen Effekt abzuschätzen, solange man keine wirkliche Theorie der geordneten Phase besitzt. Wir wollen daher rein empirisch vorgehen und die Frage stellen, ob man einen formalen Ansatz für den temperaturabhängigen Teil der Energiedichte angeben kann, der die tatsächlichen Verhältnisse befriedigend darstellt. Wir bezeichnen mit $\Delta u_{T}$ die Differenz der Energiedichten der geordneten und der ungeordneten Phase. Dieser Wert $\Delta u_{T}$ muß absolut genommen größer sein als $u$ in Gl. (40), da die mittlere Energiedichte der ungeordneten Phase mit wachsender Temperatur steigt, und zwar proportional zu $T^{2}$. Ferner soll der Ausdruck $\left(1-e^{-4 a}\right) / \alpha$, der ein Maß für die bedeckte Oberfläche der Impulskugel ist, mit

$$
\left(1-e^{-4 a}\right) / \alpha=O
$$

abgekürzt werden.

Der folgende Ansatz gibt ein Beispiel, das die tatsächlichen Verhältnisse recht befriedigend darstellt:

$$
\begin{aligned}
\Delta u_{T}= & \frac{h^{3}}{2 \pi P m} \frac{\Delta n^{2}}{O}\left[1-\frac{1}{Z} \lg \frac{2 \pi P^{3} O}{\Delta n h^{3}}\right] \\
& -\frac{4 \pi P m}{h^{3}}(k T)^{2}\left(\delta O+\varepsilon O^{2}+\ldots\right) .
\end{aligned}
$$

Hierin sollen $\delta$ und $\varepsilon$ Zahlenkoeffizienten der Größenordnung 1 sein. Anschaulich bedeutet der Ansatz (48), daß die Stellen auf der Oberfläche der Impulskugel, an denen es Wellenpakete der beschriebenen Art gibt, nicht mehr zur spezifischen Energie der Elektronen beitragen, und zwar unabhängig von der Dichte der Wellenpakete. Wir müssen nun zunächst die zugehörige Differenz 
der freien Energien (richtiger: Energiedichten) berechnen. Wenn man annimmt, daß die freie Energie von $T, O$ und $\Delta n$ in der Form

$$
\Delta f=-\varphi(O, \Delta n)+T^{2} \chi(O, \Delta n)
$$

abhängt, so würde für die Differenz der Entropien $S$ gelten

$$
\begin{aligned}
\Delta S & =-\frac{d \Delta f}{d T} \\
& =-\frac{\partial \Delta f}{\partial T}-\frac{\partial \Delta f}{\partial O} \frac{d O}{d T}-\frac{\partial \Delta f}{\partial \Delta n} \frac{d \Delta n}{d T} .
\end{aligned}
$$

Da aber im thermodynamischen Gleichgewicht

$$
\partial \Delta f / \partial O=0 \quad \text { und } \quad \partial \Delta f / \partial \Delta n=0
$$

sein müssen, wird

$$
\Delta S=-\partial \Delta f / \partial T=-2 T \chi(O, \Delta n) .
$$

und $\Delta u=\Delta f+T \Delta S$

$$
=-\varphi(O, \Delta n)-T^{2} \chi(O, \Delta n) \text {. }
$$

Der Vergleich von (48), (49) und (53) gibt daher umgekehrt

$$
\begin{array}{r}
\Delta f=\frac{h^{3}}{2 \pi P m} \frac{\Delta n^{2}}{O}\left[1-\frac{1}{Z} \lg \frac{2 \pi P^{3} O}{h^{3} \Delta n}\right] \\
+\frac{4 \pi P m}{h^{3}}-(k T)^{2}\left(\delta O+\varepsilon O^{2}\right) .
\end{array}
$$

Im thermodynamischen Gleichgewicht muß die freie Energie als Funktion von $\Delta n$ und $O$ ein Minimum sein. Man erhält also nach (51) :

$$
\begin{aligned}
\frac{\partial \Delta f}{\partial \Delta n} & =\frac{h^{3}}{\pi P m} \frac{\Delta n}{O}\left[1-\frac{1}{Z} \lg \frac{2 \pi P^{3} O}{\Delta n h^{3}}+\frac{1}{2 Z}\right] \\
& =0
\end{aligned}
$$

d. h. wie in (42)

$$
\Delta n=\frac{2 \pi P^{3}}{h^{3}} O e^{-Z-1 / 2} .
$$

Durch Einsetzen in $\Delta f$ ergibt sich:

$$
\begin{aligned}
\Delta f=- & \frac{\pi P^{5}}{m h^{3} Z} O e^{-2 Z-1} \\
& +\frac{4 \pi P m}{h^{3}}(k T)^{2}\left(\delta O+\varepsilon O^{2}+\ldots\right)
\end{aligned}
$$

und aus $\partial \Delta f / \partial O=0$ folgt:

$$
O=\frac{1}{2 \varepsilon}\left[\left(\frac{P^{2}}{2 m k T}\right)^{2} \frac{e^{-2 Z-1}}{Z}-\delta\right] .
$$

Allerdings kann $O$ nach (47) nicht größer als 4 werden, für tiefere Temperaturen wird also einfach $O=4$.

$$
\begin{aligned}
& \Delta f=-\frac{\pi m P}{\varepsilon h^{3}}(k T)^{2} \\
& {\left[\left(\frac{P^{2}}{2 m k T}\right)^{2} \frac{e^{-2 Z-1}}{Z}-\delta\right]^{2} \text { für } O \leqslant 4,}
\end{aligned}
$$

$$
\begin{aligned}
\Delta f=-\frac{4 \pi P^{5}}{m h^{3} Z} e^{-2 Z-1} & \\
& +\frac{4 \pi P m}{h^{3}}(k T)^{2}(4 \delta+16 \varepsilon)
\end{aligned}
$$

für tiefere Temperaturen.

Aus der Differenz der freien Energien kann man, wie es Gorter zuerst getan hat, die Temperatur des Sprungpunktes als Funktion eines etwa vorhandenen äußeren Magnetfeldes berechnen. Wenn sich nämlich der Supraleiter in einem Magnetfeld $H$ befindet, so muß die Differenz der freien Energien gleich der Arbeit sein, die der Maxwellsche Druck bei einer Verschiebung der Phasengrenze zwischen dem supraleitenden und dem nicht supraleitenden Zustand leistet. Daraus folgt nach Gorter :

$$
\begin{aligned}
\frac{1}{8 \pi} H^{2}= & \frac{\pi m P}{\varepsilon h^{3}}(k T)^{2} \\
& {\left[\left(\frac{P^{2}}{2 m k T}\right)^{2} \frac{e^{-2 Z-1}}{Z}-\delta\right]^{2}, }
\end{aligned}
$$

d. h.

$$
\begin{aligned}
& H=\pi \sqrt{\frac{8 P m}{h^{3} \varepsilon} k T} \\
& {\left[\left(\frac{P^{2}}{2 m k T}\right)^{2} \frac{e^{-2 Z-1}}{Z}-\delta\right] }
\end{aligned}
$$

für $O \leqslant 4$ oder, was das gleiche ist, für

$$
T \geqslant T_{1}=\frac{P^{2}}{2 k m} \frac{e^{-Z-1 / 2}}{\sqrt{Z(\delta+8 \varepsilon)}} ;
$$

entsprechend

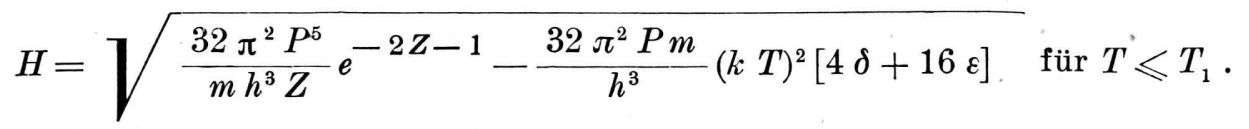


Bei der eben definierten Grenztemperatur $T_{1}$ sind $H$ und $d H / T$ stetig, $d^{2} H / d T^{2}$ ändert sich dort unstetig von negativen $\mathrm{zu}$ positiven Werten; physikalisch bedeutet $T_{1}$ diejenige Temperatur, bei der zum erstenmal der geordnete Zustand ohne Strom $(\alpha=0)$ der thermodynamisch stabilste $\mathrm{Zu}$ stand wird, während zwischen $T_{1}$ und dem Sprungpunkt $T_{0}$ der thermodynamisch stabilste Zustand einen Strom besitzt. Der Sprungpunkt $T_{0}$ (ohne Magnetfeld) liegt nach (61) bei

$$
T_{0}=\frac{P^{2}}{2 k m} \frac{e^{-Z-1 / 2}}{\sqrt{Z \delta}} .
$$

Der ganze Verlauf der Kurve $H(T)$ ist für den speziellen Wert $\epsilon=\delta / 8$, d. h. $T_{1}=T_{0} / \sqrt{2}$ in Abb. 2 graphisch dargestellt. Man erkennt, daß man tatsächlich die empirischen Verhältnisse zum mindesten qualitativ gut darstellen kann. Es muß aber nochmal betont werden, daß Gl. (48) hinsichtlich der zu $T^{2}$ proportionalen Glieder ein willkürlicher, ad hoc angeschriebener Ansatz, nicht das Ergebnis einer Theorie ist. Von physikalischen Utberlegungen ausgehend, hätte man vielleicht eine etwas andere Abhängigkeit der zu $T^{2}$ proportionalen Glieder von $O$ und $\Delta n$ erwartet; insbesondere müßte man wohl annehmen, daß diese Glieder nicht nur von $O$, sondern auch von $\Delta n$ abhängen; denn wenn die Dichte der geordneten Phase sehr klein wird, kann sie die thermische Bewegung der Elektronen in der ungeordneten Phase nicht mehr erheblich stören. Im ganzen reichen eben die Ansätze des Kap. 2 zu einer quantitativen Behandlung des Problems nicht aus. In Wirklichkeit wird schon die Wellenfunktion der geordneten Phase (also z. B. die Form des Wellenpakets) von der Wechselwirkung mit der ungeordneten Phase und damit indirekt von der Temperatur abhängen.

\section{Die elektrodynamischen Gleichungen}

a) Die Rechnungen des letzten Abschnitts haben gezeigt, daß für einen bestimmten Temperaturbereich die Entstehung eines geordneten Zustandes mit Strom energetisch günstiger sein kann als die eines Zustandes ohne Strom. Aber selbst bei tieferen Temperaturen $\left(T \leqslant T_{1}\right)$, wo der Zustand ohne Strom der thermodynamisch stabilste ist, wird sich bei Vorhandensein eines geeigneten „Kristallkeims“ häufig ein Zustand mit Strom herausbilden, der dann auch nicht mehr durch Wechselwirkung mit dem Ionengitter in einen Zustand ohne Strom übergehen kann. Im

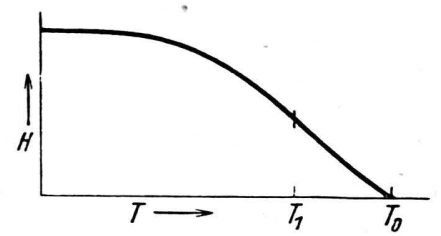

Abb. 2. Kritisches Magnetfeld in Abhängigkeit von der Temperatur.

allgemeinen wird es also in einem Supraleiter, auch wenn er makroskopisch stromlos ist, statistisch verteilte Stromfäden geben, deren Stromdichte nach (46) und (56) die Größenordnung

$$
s \sim \frac{e P^{4}}{m h^{3}} e^{-Z-1 / 2}\left(\sim 10^{8} \mathrm{Amp} / \mathrm{cm}^{2}\right)
$$

hat. Diese Stromfäden werden eine ähnliche Rolle spielen wie die Wei ßschen Bezirke beim Ferromagneten. Wir betrachten nun ein Gebiet, das kleiner ist als diese Bezirke, und fragen, wie sich dort ein etwa vorhandener Strom zeitlich verändert. Die Antwort wird qualitativ etwa folgendermaßen lauten: Eine Stromdichte, die größer ist als (65), wird durch die Zusammenstöße der Elektronen mit dem Ionengitter abnehmen, bis ein Wert der Ordnung (65) erreicht ist, dann wird sie konstant bleiben. Oder anschaulich: Wenn durch ein äußeres Feld das Fermi-Gas in Bewegung gesetzt worden ist, so wird es sich durch die Zusammenstöße mit den Ionen ,abkühlen“ und schließlich an irgendeinem „Kristallkeim“ in der geordneten Phase ausfrieren. Dieses Verhalten kann man qualitativ etwa durch eine Gleichung von dem Typus

$$
\lambda \dot{\mathfrak{S}}=-\frac{\mathfrak{g}}{\sigma} \frac{s-s_{0}}{s}
$$

darstellẹn, worin $\mathfrak{g}$ die Stromdichte im Bezirk, $\sigma$ die gewöhnliche Leitfähigkeit und $s_{0}$ einen Betrag der Größenordnung (65) bedeutet. Wenn ein äußeres Feld (₹ vorhanden ist, so tritt an die Stelle von $(66)$ :

$$
\lambda \dot{\mathfrak{G}}=\mathfrak{F}-\frac{\mathfrak{g}}{\sigma} \frac{s-s_{0}}{s} .
$$

In den beiden Gleichungen ist $\lambda$ ein $\mathrm{Maß}$ für die Trägheit der Elektronen und hat den Wert 


$$
\lambda=m / n e^{2},
$$

wobei $n$ die Anzahl der Leitungselektronen pro Volumeneinheit und $m$ ihre effektive Masse bedeutet. Wenn nun ursprünglich die Stromdichte $\mathfrak{g}_{0}$ vorhanden war, so werden bei normalen elektrischen Feldern die Abweichungen der Stromdichte von $\mathfrak{S}_{0}$ dauernd sehr klein bleiben. Man setzt daher zweckmäßig $\mathfrak{S}=\mathfrak{S}_{0}+\mathscr{A} \mathfrak{I}$ und betrachtet getrennt die Komponenten von $\Delta \mathfrak{S}$ parallel und senkrecht zu $\mathfrak{S}_{0}$. Dann gilt in hinreichender Näherung:

$$
\left.\begin{array}{l}
\lambda \Delta \dot{\mathfrak{B}}_{\|}=\mathfrak{E}_{\|}-\frac{\Delta \mathfrak{I}_{\|}}{\sigma}, \\
\lambda \Delta \dot{\mathfrak{g}}_{\perp}=\mathfrak{E}_{\perp} .
\end{array}\right\}
$$

Um die makroskopische Stromdichte zu bekommen, muß man jetzt noch über die verschiedenen Stromfäden mit verschiedenen Richtungen und Werten von $\mathfrak{g}_{0}$ mitteln. Die Beiträge zur makroskopischen Stromdichte $\mathfrak{i}$, die von $\mathcal{A \mathfrak { F }}_{\|}$herrühren, wollen wir $i_{n}$ nennen, die Beiträge, die von $\Delta^{\mathfrak{S}_{\perp}}$ herrühren, $\mathfrak{i}_{s} ;$ also $\mathfrak{i}_{n}=\overline{\Delta \mathfrak{S}}_{\|} ; \mathfrak{i}_{s}=\overline{\Delta \mathfrak{S}}_{\perp}$. Da alle Richtungen von $\mathfrak{g}_{0}$ gleich wahrscheinlich sind, wird

$$
\overline{\mathfrak{F}}_{\|}=\frac{1}{3} \mathfrak{\xi} \text { und } \overline{\mathfrak{F}}_{\perp}=\frac{2}{3} \mathfrak{\xi} \text {. }
$$

So ergibt sich schließlich:

$$
\begin{aligned}
& \lambda d \dot{i}_{n} / d t=\frac{1}{3} \xi-\frac{\mathfrak{i}_{n}}{\sigma} . \\
& \lambda d \dot{\mathfrak{i}}_{s} / d t=\frac{2}{3} \xi .
\end{aligned}
$$

Diese Gleichungen berechtigen uns, $i_{n}$ als die normále Stromdichte, $\mathfrak{i}_{s}$ als die Supraleitungsstromdichte $\mathrm{zu}$ bezeichnen. Die zweite der Gl. (70)

$$
d \mathfrak{i}_{s} / d t=\frac{2}{3 \hat{\lambda}} \mathfrak{F}
$$

unterscheidet sich von der entsprechenden Londonschen Gleichung nur durch den Faktor von ₹, der hier den Wert $2 n e^{2} / 3 m$ hat, während er bei London $n e^{2} / m$ beträgt, wobei $n$ aber bei Lond on die Anzahl der Supraleitungselektronen bedeuten soll. Die empirischen Werte des Faktors

7 Vergl. dazu auch frühere Arbeiten, z. B. R.B e c k e r, G. Heller u. F. Sa uter, Z. Physik 85, 772 [1933].

8 W. Meissner u. R. Ochsenfeld, Naturwiss. 21, 787 [1933]; Z. techn. Physik 15, 507 [1934]. von $\mathfrak{5}$ in (71), die aus den Versuchen über die Eindringtiefe eines Magnetfeldes in Supraleiter erschlossen werden können, sprechen eher für Werte von $n$ in der Größenordnung der Anzahl aller Leitungselektronen. An der Supraleitung können sich aber nach Ausweis der tiefen Sprungtemperaturen und kleinen Umwandlungswärmen kaum mehr Elektronen beteiligen als an der spezifischen Wärme, d. h. nur etwa der $1^{-4}$. Teil aller Leitungselektronen. Die Zahl $n$ im Faktor $\lambda$ kann also kaum etwas mit der Anzahl der Elektronen in der geordneten Phase zu tun haben, sondern er bedeutet die Zahl aller Leitungselektronen; der Faktor $2 / 3$ kann wegen der Unsicherheit der experimentellen Daten und ihrer Auswertung wohl einstweilen nicht an der Erfahrung geprüft werden.

Dagegen zeigen die Experimente eindeutig eine Zunahme von $\lambda$, d. h. eine Zunahme der Eindringtiefe $^{7}$ mit Annäherung an den Sprungpunkt. $\lambda$ scheint bei $T=T_{0}$ unendlich zu' werden. Diese Zunahme wird durch Gl. (68) nicht wiedergegeben. Es ist aber wahrscheinlich, daß eine genauere Behandlung der Verhältnisse in der Nähe von $T_{0}$ die Gln. (68) und (70) durch einen komplizierteren Zusammenhang ersetzen würde. Denn in der Nähe von $T_{0}$ scheinen $O$ und $\Delta n$ nach (58) zu verschwinden; die Supraleitungsströme müssen also schon deswegen eine dickere Oberflächenschicht erfüllen (und das Magnetfeld tiefer eindringen), weil die Dichte $\Delta n$ der Supraleitungselektronen immer kleiner, ihr Abstand in der geordneten Phase immer größer wird; und man wird wohl annehmen müssen, daß die Eindringtiefe mindestens, sagen wir, dreimal so groß sein muß wie der Gitterabstand des Elektronengitters. Die Gln. (68) und (70) gelten also nur in hinreichendem Abstand unter dem Sprungpunkt $T_{0}$ und besagen, daß in diesem tieferen Temperaturgebiet $\lambda$ konstant sein sollte, was an der Erfahrung geprüft werden kann.

b) Gl. (71) enthält bekanntlich noch nicht die Beobachtung von W. M eissner und R. Ochsenfeld ${ }^{8}$, daß bei der Abkühlung eines Körpers im Magnetfeld unter den Sprungpunkt Oberflächenströme entstehen, die das Magnetfeld aus dem Supraleiter verdrängen. Um diesen Sachverhalt zu erklären, betrachten wir die Energiebilanz in einem Supraleiter unter Benützung von (70), wobei wir der Einfachheit halber $\varepsilon=\mu=1$ annehmen: 


$$
\begin{aligned}
\frac{d}{d t} \int d V \frac{1}{8 \pi}\left(\mathfrak{F}^{2}+\mathfrak{S}^{2}\right) & =-\frac{c}{4 \pi} \int d f[\mathfrak{F} \mathfrak{S}]_{n}-\int d V \mathfrak{F}\left(i_{n}+i_{s}\right) \\
& =-\frac{c}{4 \pi} \int d f[\mathfrak{F}]_{n}-\int d V\left(3 \lambda_{i_{n}} i_{n}+\frac{3 i_{n}{ }^{2}}{\sigma}+\frac{3}{2} \lambda i_{s} i_{s}\right) .
\end{aligned}
$$

Dieses Ergebnis kann auch in der Form ausgesprochen werden

$$
\frac{d}{d t} \int d V\left[\frac{1}{8 \pi}\left(\mathfrak{E}^{2}+\mathfrak{S}^{2}\right)+\frac{3}{2} \lambda i_{n}{ }^{2}+\frac{3}{4} \lambda i_{s}{ }^{2}\right]=-\frac{c}{4 \pi} \int d f[\mathfrak{F} \mathfrak{S}]_{n}-\int d V \frac{3 i_{n}{ }^{2}}{\sigma} .
$$

Abgesehen von dem im Poyntingschen Vektor ausgedrückten Energiestrom durch die Oberfläche kann also der Ausdruck auf der linken Seite von (73) durch die Joulesche Wärme nur abnehmen. Wenn nun ein Supraleiter im konstanten äußeren Magnetfeld unter den Sprungpunkt abgekühlt wird, so entstehen zunächst durch die Bildung der geordneten Phase lokale Ströme, die zu lokalen magnetischen Feldern und, da sie zeitlich veränderlich sind, zu lokalen, zeitlich veränderlichen elektrischen Feldern Anlaß geben. Dieser Prozeß wird so lange fortgehen, bis der Ausdruck auf der linken Seite von (73) seinen Minimalwert (unter den gegebenen Bedingungen) erreicht hat. Im Endzustand sind $\mathfrak{F}$ und $\mathfrak{i}_{\boldsymbol{s}}$ zeitlich konstant, $\mathfrak{E}$ und $\mathfrak{i}_{n}$ verschwinden. Es muß also

$$
\int d V\left[\frac{1}{8 \pi} \mathfrak{S}^{2}+\frac{3}{4} \lambda i_{s}{ }^{2}\right]=\operatorname{Min}
$$

gefordert werden, wobei die Maxwellsche Gleichung $\operatorname{rot} \mathfrak{S}=4 \pi \mathfrak{i} / c$ zu berücksichtigen ist. Die Variation gibt:

$$
\begin{aligned}
& \int d V\left[\frac{1}{4 \pi} \mathfrak{H} \delta \mathfrak{S C}+\frac{3}{2} \lambda i_{s} \delta i_{s}\right] \\
& =\int d V\left[\frac{1}{4 \pi} \mathfrak{H} \delta \mathfrak{S}+\frac{3}{2} \lambda i_{s} \frac{c}{4 \pi} \operatorname{rot} \delta \mathfrak{S}\right] \quad \\
& =\int \frac{d V}{4 \pi} \delta \mathfrak{S}\left[\mathfrak{S}+\frac{3}{2} \lambda c \operatorname{rot} i_{s}\right]+\int d f\left[\delta \mathfrak{H} i_{s}\right]_{n} \\
& =0 .
\end{aligned}
$$

Da das Oberflächenintegral nichts beiträgt, wenn man die Oberfläche um das Metallstück herumlegt, so daß dort $\dot{i}_{s}$ verschwindet, so folgt

$$
\begin{gathered}
\mathfrak{H}+\frac{3}{2} \lambda c \operatorname{rot} \mathfrak{i}_{s}=0 \text { oder } \\
\operatorname{rot} \dot{\mathfrak{i}}_{s}=-\frac{2}{3 \lambda c} \mathfrak{S} .
\end{gathered}
$$

Diese Gleichung, die zuerst von London an- gegeben worden ist, beschreibt, soviel wir wissen, die Verhältnisse beim Meissner-Ochsenfeldschen Versuch richtig. Sie gilt nicht nur für konstante, sondern auch für zeitlich veränderliche Felder $\mathfrak{S}$, da aus Gl. (71) bereits folgt

$$
\operatorname{rot} \ddot{\mathfrak{i}}_{s}=-\frac{2}{3 \lambda c} \dot{\mathfrak{S}} \text {. }
$$

Der Meissner-Ochsenfeld-Effekt wird hier also thermodynamisch auf Grund des zweiten Hauptsatzes erklärt: durch die Feldschwankungen beim Übergang in die geordnete Phase wird so viel Feldenergie in $\mathrm{J}$ o u le sche Wärme umgesetzt, wie unter den gegebenen Bedingungen umgesetzt werden kann. Das System stellt sich auf das Minimum der freien Energie ein.

Bei den Rechnungen dieses Abschnitts wurde angenommen, daß die elektrischen Felder im Supraleiter stets so gering sind, daß die Abweichungen $\mathscr{A} \mathfrak{Z}$ der Stromdichte im einzelnen Stromfaden vom Wert $\mathfrak{I}_{0}$ der ursprünglichen Supraleitungsstromdichte stets sehr klein bleiben: $|\Delta \mathfrak{F}| \ll|\mathfrak{F}|$. In diesem Fall wird wegen der statistischen Verteilung der Stromfäden auch die Gesamtstromdichte $|\mathfrak{i}| \ll\left|\mathfrak{S}_{0}\right|$ sein. Es entsteht nun die Frage, ob nicht doch auch Verhältnisse hergestellt werden können, bei denen alle Stromfäden praktisch parallel laufen; d. h. $\mathfrak{i}_{s} \approx \mathfrak{g}_{0}$. In solchen Fällen wären die Gln. (70) nicht mehr richtig. Es würden Sättigungserscheinungen eintreten, die eine Steigerung von $\mathfrak{i}_{s}$ über $\mathfrak{g}_{0}$ hinaus verhindern.

Diese Frage kann man beantworten, indem man die Stromdichte $\mathfrak{g}_{0}$, deren Größenordnung durch (65) gegeben ist, mit der größten Stromdichte vergleicht, die in einem Supraleiter bei Annahme der Gln. (48) bis (63) und (70) möglich ist. Schon durch die Gln. (48) bis (63) und (70), (76) ist ja die Stromdichte nach oben begrenzt, da ein hoher Strom in seiner Umgebung hohe Magnetfelder erzeugt und hohe Magnetfelder die Supraleitung zerstören. 
Zunächst soll die maximale Stromdichte, die durch (48) bis (63) und (70), (76) vorgeschrieben wird, berechnet werden. Dabei betrachten wir der Einfachheit halber die Verhältnisse am absoluten Nullpunkt $(T=0)$ und nehmen an, es handle sich um den Strom in einem geradlinigen Supraleiter von kreisförmigem Querschnitt (Radius $R$ ). Wegen der im stationären Falle gültigen Beziehungen:

$$
\begin{gathered}
\operatorname{rot} \mathfrak{H}=\frac{4 \pi \dot{i}_{s}}{c} ; \operatorname{rot} \mathfrak{i}_{s}=-\frac{2}{3 \lambda c} \mathfrak{S} \\
\operatorname{rot} \operatorname{rot} \dot{\mathfrak{i}}_{s}=-\frac{8 \pi}{3 c^{2} \lambda_{s}} \mathfrak{i}_{s}=-\frac{\mathfrak{i}_{s}}{l^{2}}, \\
\operatorname{oder} \Delta \mathfrak{i}_{s}=\dot{i}_{s} / l^{2},
\end{gathered}
$$

wobei $l=\sqrt{3 c^{2} \lambda / 8 \pi}=\sqrt{3 c^{2} m / 8 \pi n e^{2}}$ die „Eindringtiefe" des Supraleiters bedeutet. Wenn wir $l \ll R$ voraussetzen, so wird

$$
\mathrm{i}_{s} \approx \mathfrak{i}_{s o} e^{(r-R) / l} \text { und } I \approx 2 \pi R l \mathfrak{i}_{s o} .
$$

Das Magnetfeld an der Oberfläche des Drahtes wird daher:

$$
H=\frac{2 I}{c R}=\frac{4 \pi}{c} l \mathfrak{i}_{s o} .
$$

Die maximale Stromdichte erhält man dann, indem man in (80) für $H$ das größte für $T=0$ mögliche Magnetfeld einsetzt, das die Supraleitung noch nicht zerstört:

$$
i_{s \max }=\frac{c}{4 \pi l} H_{\max } .
$$

Dieses maximale Feld $H$ hängt nach (57) wegen
$\Delta f=\frac{1}{8 \pi} H_{\max }^{2}$ noch von dem $\alpha$ - bzw. 0-Wert der Stromfäden ab, die hier die Supraleitung erzeugen:

$$
H_{\max }=\sqrt{\frac{3 \pi n P^{2}}{m Z}} \sqrt{O} e^{-Z-1 / 2}
$$

und

$$
i_{s \max }=n \frac{P e}{m} \sqrt{\frac{O}{2 Z}} e^{-Z-1 / 2} .
$$

Dieser Wert ist unter Berücksichtigung von (46) und (56) zu vergleichen mit der Stromdichte im einzelnen Stromfaden:

$s_{0}=\frac{3}{4} n \frac{P e}{m} O e^{-Z-1 / 2}\left(\operatorname{stg} 2 a-\frac{1}{2 a}\right)$.

Man erkennt sofort, daß die beiden Ausdrücke von der gleichen Größenordnung sind, da bei $T=0$ für $O$ und $\propto$ Werte der Größenordnung 1 am häufigsten vorkommen werden.

Aus diesem Ergebnis folgt: Wenn man in einem Supraleiter die höchsten Ströme erzeugt, die eben die Supraleitung noch nicht zerstören, so sind - wenigstens bei sehr tiefen Temperaturen die einzelnen Stromfäden schon zu einem erheblichen Teil parallel gerichtet. Daher muß man erwarten, daß bei diesen höchsten Stromdichten schon erhebliche Abweichungen von den üblichen Gln. (70) und (76) der Londonschen Theorie auftreten. 